\title{
An extension of the univalence criterion for a family of integral operators
}

\begin{abstract}
The main object of the present paper is to extend the univalence condition for a family of integral operators. Relevant connections of some of the results obtained in this paper with those in earlier works are also provided.
\end{abstract}

1. Introduction and preliminaries. Let $\mathcal{A}$ denote the class of functions $f$ normalized by

$$
f(z)=z+\sum_{n=2}^{\infty} a_{n} z^{n}
$$

which are analytic in the open unit disk $\mathcal{U}=\{z: z \in \mathbb{C}$ and $|z|<1\}$ and satisfy the conditions $f(0)=f^{\prime}(0)-1=0$.

Consider $\mathcal{S}=\{f \in \mathcal{A}: f$ is a univalent function in $\mathcal{U}\}$. if

A function $f(z) \in \mathcal{A}$ is said to be a member of the class $\mathcal{B}(\gamma)$ if and only

$$
\left|\frac{z^{2} f^{\prime}(z)}{f^{2}(z)}-1\right|<1-\gamma, \quad 0 \leq \gamma<1
$$

Recently, Frasin and Darus (see [6]) defined and studied the class $\mathcal{B}(\gamma)$. In his paper Frasin (see [4]) obtained some results for functions belonging

2000 Mathematics Subject Classification. 30C45.

Key words and phrases. Integral operator, analytic functions, univalent functions, open unit disk, univalence criterion. 
to this class and also he showed that if $f(z) \in \mathcal{B}(\gamma)$ then $f(z)$ satisfies the following inequality

$$
\left|\frac{z f^{\prime \prime}(z)}{f^{\prime}(z)}\right| \leq \frac{(1-\gamma)(2+|z|)}{1-|z|} \quad(z \in \mathcal{U})
$$

For $\gamma=0$ the class $\mathcal{B}(0)=\mathcal{T}$ was studied by Ozaki and Nunokawa (see $[8]$ ).

We denote by $\mathcal{W}$ the class of functions $w$ which are analytic in $\mathcal{U}$ satisfying the conditions $|w(z)|<1$ and $w(0)=w^{\prime}(0)=0$ for all $z \in \mathcal{U}$.

Now, by Schwarz's lemma, it follows that

$$
|w(z)|<|z| .
$$

In [7], we see that if $w(z) \in \mathcal{W}$, then $w(z)$ satisfies

$$
\left|w^{\prime}(z)\right| \leq \frac{1-|w(z)|^{2}}{1-|z|^{2}} \quad(z \in \mathcal{U}) .
$$

In [11], N. Seenivasagan and D. Breaz considered the following family of integral operators $\mathcal{F}_{\alpha_{1}, \alpha_{2}, \ldots, \alpha_{n}, \beta}(z)$ defined as follows

$$
\mathcal{F}_{\alpha_{1}, \alpha_{2}, \ldots, \alpha_{n}, \beta}(z):=\left(\beta \int_{0}^{z} t^{\beta-1} \prod_{i=1}^{n}\left(\frac{f_{i}(t)}{t}\right)^{\frac{1}{\alpha_{i}}} d t\right)^{\frac{1}{\beta}},
$$

where $f_{i} \in \mathcal{A}, f_{i}^{\prime \prime}(0)=0$ and $\alpha_{1}, \alpha_{2}, \ldots, \alpha_{n}, \beta \in \mathbb{C}$ for all $i \in\{1,2, \ldots, n\}$.

When $\alpha_{i}=\alpha$ for all $i \in\{1,2, \ldots, n\}, \mathcal{F}_{\alpha_{1}, \alpha_{2}, \ldots, \alpha_{n}, \beta}(z)$ becomes the integral operator $\mathcal{F}_{\alpha, \beta}(z)$ considered in (see [1]).

We begin by recalling each of the following theorems dealing with univalence criterion, which will be required in our present paper.

In [10], Pascu proved the following theorem.

Theorem 1 (Pascu [10]). Let $\beta$ be a complex number with $\operatorname{Re}(\beta)>0$ and $f \in \mathcal{A}$. If

$$
\frac{1-|z|^{2 \operatorname{Re}(\beta)}}{\operatorname{Re}(\beta)}\left|\frac{z f^{\prime \prime}(z)}{f^{\prime}(z)}\right| \leq 1
$$

for $z \in \mathcal{U}$, then the function

$$
F_{\beta}(z):=\left(\beta \int_{0}^{z} t^{\beta-1} f^{\prime}(t) d t\right)^{\frac{1}{\beta}}
$$

belongs to $\mathcal{S}$.

In [9], Pascu and Pescar obtained the next result. 
Theorem 2 (Pascu and Pescar [9]). Let $\beta$ and $\mu$ be complex numbers, and $g \in \mathcal{S}$. If $\operatorname{Re}(\beta)>0$ and $|\mu| \leq \min \left\{\frac{1}{2} \operatorname{Re}(\beta) ; \frac{1}{4}\right\}$, then the function

$$
\mathcal{G}_{\beta, \mu}(z):=\left(\beta \int_{0}^{z} t^{\beta-1}\left(\frac{g(t)}{t}\right)^{\mu} d t\right)^{\frac{1}{\beta}}
$$

belongs to $\mathcal{S}$.

Note that Theorem 2 includes the special case of Pascu and Pescar's theorem (see [9]) when $\operatorname{Re}(\alpha)=\operatorname{Re}(\beta)$.

In the present paper, we propose to investigate further univalence condition involving the general a family of integral operators defined by (1.6).

2. Main results. In this section, we first state an inclusion for $f(z) \in$ $\mathcal{B}(\gamma)$, then we give the main univalence condition involving the general integral operator given by (1.6).

Theorem 3. If $f(z) \in \mathcal{B}(\gamma)$, then the inequality is satisfied

$$
\left|\frac{z f^{\prime}(z)}{f(z)}-1\right| \leq \frac{(1-\gamma)(1+|z|)}{1-|z|}
$$

for all $z \in \mathcal{U}$.

Proof. Let $f(z) \in \mathcal{B}(\gamma)$. Then we have

$$
\frac{z^{2} f^{\prime}(z)}{f^{2}(z)}=1+(1-\gamma) w(z)
$$

where $w(z) \in \mathcal{W}$. By applying the logarithmic differentiation, we obtain from (2.2) that

$$
\frac{z f^{\prime \prime}(z)}{f^{\prime}(z)}=\frac{(1-\gamma) z w^{\prime}(z)}{1+(1-\gamma) w(z)}+\frac{2 z f^{\prime}(z)}{f(z)}-2
$$

and

$$
\frac{z f^{\prime}(z)}{f(z)}-1=\frac{1}{2}\left(\frac{z f^{\prime \prime}(z)}{f^{\prime}(z)}-\frac{(1-\gamma) z w^{\prime}(z)}{1+(1-\gamma) w(z)}\right),
$$

thereby, it follows that

$$
\begin{aligned}
\left|\frac{z f^{\prime}(z)}{f(z)}-1\right| & =\left|\frac{1}{2}\left(\frac{z f^{\prime \prime}(z)}{f^{\prime}(z)}-\frac{(1-\gamma) z w^{\prime}(z)}{1+(1-\gamma) w(z)}\right)\right| \\
& \leq \frac{1}{2}\left(\left|\frac{z f^{\prime \prime}(z)}{f^{\prime}(z)}\right|+\left|\frac{(1-\gamma) z w^{\prime}(z)}{1+(1-\gamma) w(z)}\right|\right) \\
& \leq \frac{1}{2}\left(\left|\frac{z f^{\prime \prime}(z)}{f^{\prime}(z)}\right|+\frac{(1-\gamma)|z|\left|w^{\prime}(z)\right|}{1-(1-\gamma)|w(z)|}\right) .
\end{aligned}
$$


From (1.3) and (1.5), we have

$$
\left|\frac{z f^{\prime}(z)}{f(z)}-1\right| \leq \frac{1}{2}\left(\frac{(1-\gamma)(2+|z|)}{1-|z|}+\frac{(1-\gamma)|z|}{1-(1-\gamma)|w(z)|} \frac{1-|w(z)|^{2}}{1-|z|^{2}}\right)
$$

and for $0 \leq \gamma<1$, it is easy to show that

$$
\frac{1-|w(z)|}{1-(1-\gamma)|w(z)|} \leq 1 \quad(z \in \mathcal{U}) .
$$

From (1.4), (2.3) and (2.4), we obtain that

$$
\left|\frac{z f^{\prime}(z)}{f(z)}-1\right| \leq \frac{(1-\gamma)(1+|z|)}{1-|z|}
$$

This evidently completes the proof of Theorem 3 .

Next we prove the following main theorem.

Theorem 4. Let $f_{i}(z) \in \mathcal{B}(\gamma)$ for $i \in\{1,2, \ldots, n\}$. Let $\beta$ be a complex number with $\operatorname{Re}(\beta)>0$. If

$$
\sum_{i=1}^{n} \frac{1}{\left|\alpha_{i}\right|} \leq \min \left\{\frac{1}{2(1-\gamma)} \operatorname{Re} \beta ; \frac{1}{4(1-\gamma)}\right\}
$$

for all $z \in \mathcal{U}$, then the function

$$
\mathcal{F}_{\alpha_{1}, \alpha_{2}, \ldots, \alpha_{n}, \beta}(z):=\left(\beta \int_{0}^{z} t^{\beta-1} \prod_{i=1}^{n}\left(\frac{f_{i}(t)}{t}\right)^{\frac{1}{\alpha_{i}}} d t\right)^{\frac{1}{\beta}}
$$

belongs to $\mathcal{S}$.

Proof. Define function

$$
h(z)=\int_{0}^{z} \prod_{i=1}^{n}\left(\frac{f_{i}(t)}{t}\right)^{\frac{1}{\alpha_{i}}} d t .
$$

We have $h(0)=h^{\prime}(0)-1=0$. Also, a simple computation yields

$$
h^{\prime}(z)=\prod_{i=1}^{n}\left(\frac{f_{i}(z)}{z}\right)^{\frac{1}{\alpha_{i}}} .
$$

Making use of logarithmic differentiation in (2.7), we obtain

$$
\frac{z h^{\prime \prime}(z)}{h^{\prime}(z)}=\sum_{i=1}^{n} \frac{1}{\alpha_{i}}\left(\frac{z f_{i}^{\prime}(z)}{f_{i}(z)}-1\right) \text {. }
$$

We thus have from (2.8) that

$$
\left|\frac{z h^{\prime \prime}(z)}{h^{\prime}(z)}\right| \leq \sum_{i=1}^{n} \frac{1}{\left|\alpha_{i}\right|}\left|\frac{z f_{i}^{\prime}(z)}{f_{i}(z)}-1\right| \text {. }
$$


By using the Theorem 3, we get the inequality

$$
\left|\frac{z h^{\prime \prime}(z)}{h^{\prime}(z)}\right| \leq \sum_{i=1}^{n} \frac{1}{\left|\alpha_{i}\right|} \frac{(1-\gamma)(1+|z|)}{1-|z|} .
$$

From (2.9), we obtain

$$
\begin{aligned}
\frac{1-|z|^{2 \operatorname{Re}(\beta)}}{\operatorname{Re}(\beta)}\left|\frac{z h^{\prime \prime}(z)}{h^{\prime}(z)}\right| & \leq \frac{1-|z|^{2 \operatorname{Re}(\beta)}}{\operatorname{Re}(\beta)} \frac{(1-\gamma)(1+|z|)}{1-|z|} \sum_{i=1}^{n} \frac{1}{\left|\alpha_{i}\right|} \\
& \leq \frac{1-|z|^{2 \operatorname{Re}(\beta)}}{1-|z|} \frac{2(1-\gamma)}{\operatorname{Re}(\beta)} \sum_{i=1}^{n} \frac{1}{\left|\alpha_{i}\right|}
\end{aligned}
$$

for all $z \in \mathcal{U}$.

Let us denote $|z|=x, x \in[0,1), \operatorname{Re}(\beta)=a>0$ and $\psi(x)=\frac{1-x^{2 a}}{1-x}$. It is easy to prove that

$$
\psi(x) \leq \begin{cases}1, & \text { if } 0<a<\frac{1}{2} \\ 2 a, & \text { if } \frac{1}{2}<a<\infty .\end{cases}
$$

From (2.6), (2.10) and (2.11), we have

$$
\begin{aligned}
\frac{1-|z|^{2 \operatorname{Re}(\beta)}}{\operatorname{Re}(\beta)}\left|\frac{z h^{\prime \prime}(z)}{h^{\prime}(z)}\right| & \leq \begin{cases}\frac{2(1-\gamma)}{\operatorname{Re}(\beta)} \sum_{i=1}^{n} \frac{1}{\left|\alpha_{i}\right|}, & \text { if } 0<\operatorname{Re}(\beta)<\frac{1}{2} \\
4(1-\gamma) \sum_{i=1}^{n} \frac{1}{\left|\alpha_{i}\right|}, & \text { if } \frac{1}{2}<\operatorname{Re}(\beta)<\infty\end{cases} \\
& \leq 1
\end{aligned}
$$

for all $z \in \mathcal{U}$.

Finally, by applying Theorem 1, we conclude that the function $\mathcal{F}_{\alpha_{1}, \alpha_{2}, \ldots, \alpha_{n}, \beta}(z)$ defined by (1.6) is in the function class $\mathcal{S}$. This evidently completes the proof of Theorem 4 .

3. Some applications of Theorem 4. In this section, we give some results of Theorem 4 .

First of all, upon setting $\alpha_{i}=\alpha$, for all $i \in\{1,2, \ldots, n\}$ in Theorem 4 , we immediately arrive at the following application of Theorem 4 .

Corollary 1. Let $f_{i}(z) \in \mathcal{B}(\gamma)$ for $i \in\{1,2, \ldots, n\}$. Let $\beta$ be a complex number with $\operatorname{Re}(\beta)>0$. If

$$
\frac{1}{|\alpha|} \leq \min \left\{\frac{1}{2 n(1-\gamma)} \operatorname{Re} \beta ; \frac{1}{4 n(1-\gamma)}\right\}
$$

holds for all $z \in \mathcal{U}$, then the function

$$
\mathcal{F}_{\alpha, \beta}(z):=\left(\beta \int_{0}^{z} t^{\beta-1} \prod_{i=1}^{n}\left(\frac{f_{i}(t)}{t}\right)^{\frac{1}{\alpha}} d t\right)^{\frac{1}{\beta}}
$$


belongs to $\mathcal{S}$.

Next we set $n=1$ in Theorem 4 , we thus obtain the following interesting consequence of Theorem 4 .

Corollary 2. Let the functions $f(z) \in \mathcal{B}(\gamma)$. Let $\beta$ be a complex number with $\operatorname{Re} \beta>0$. If

$$
\frac{1}{|\alpha|} \leq \min \left\{\frac{1}{2(1-\gamma)} \operatorname{Re} \beta ; \frac{1}{4(1-\gamma)}\right\}
$$

holds for all $z \in \mathcal{U}$, then the function

$$
\mathcal{G}_{\beta, \alpha}(z):=\left(\beta \int_{0}^{z} t^{\beta-1}\left(\frac{f(t)}{t}\right)^{\frac{1}{\alpha}} d t\right)^{\frac{1}{\beta}}
$$

belongs to $\mathcal{S}$.

\section{Remark 1.}

(i) Corollary 2 provides an extension of Theorem 2 due to Pascu and Pescar (see [9]).

(ii) If we set $\gamma=0, n=1$ and $\frac{1}{\alpha}=\mu$ in Theorem 4, we obtain Theorem 2 due to Pascu and Pescar (see [9]).

(iii) If we put $\gamma=0, \beta=1$ and $\alpha$ instead of $\frac{1}{\alpha}$ in Corollary 2, we arrive at the result by Kim and Merkes (see [5]).

Remark 2. Some authors gave similar univalence conditions by using bounded functions $f(z) \in \mathcal{A}$ in their papers, see the works (for example Breaz et al. (see [2]), Breaz et al. (see [3])). We note that the functions $f \in \mathcal{A}$ do not have to be bounded.

Acknowledgement. Authors would like to thank the referee for thoughtful comments and suggestions.

\section{REFERENCES}

[1] Breaz, D., Breaz, N., The univalent conditions for an integral operator on the classes $\mathcal{S}(p)$ and $\mathcal{T}_{2}$, J. Approx. Theory Appl. 1(2) (2005), 93-98.

[2] Breaz, D., Breaz, N., Sufficient univalence conditions for analytic functions, J. Inequal. Appl. 2007 (2007), Article ID 86493, 5 pp.

[3] Breaz, D., Breaz, N. and Srivastava, H. M., An extension of the univalent condition for a family of integral operators, Appl. Math. Lett. 22 (2009), 41-44.

[4] Frasin, B. A., A note on certain analytic and univalent functions, Southeast Asian Bull. Math. 28(5) (2004), 829-836.

[5] Kim, Y. J., Merkes, E. P., On an integral of powers of a spirallike function, Kyungpook Math. J. 12 (1972), 249-252.

[6] Frasin, B. A., Darus, M., On certain analytic univalent functions, Int. J. Math. Math. Sci. 25(5) (2001), 305-310.

[7] Nehari, Z., Conformal Mapping, Dover Publications, Inc., New York, 1975. 
[8] Ozaki, S., Nunokawa, M., The Schwarzian derivative and univalent functions, Proc. Amer. Math. Soc. 33 (1972), 392-394.

[9] Pascu, N. N., Pescar, V., On the integral operators of Kim-Merkes and of Pfaltzgraff, Mathematica (Cluj) 32(55), no. 2 (1990) 185-192.

[10] Pascu, N., An improvement of Becker's univalence criterion, Proceedings of the Commemorative Session Simion Stoilow (Braşov, 1987), 43-98, Univ. Braşov, Braşov, 1987.

[11] Seenivasagan, N., Breaz, D., Certain sufficient conditions for univalence, Gen. Math. 15(4) (2007), 7-15.

E. Deniz

Department of Mathematics

Faculty of Science

Ataturk University

Erzurum, 25240

Turkey

e-mail: edeniz@atauni.edu.tr

Received October 29, 2009
H. Orhan

Department of Mathematics

Faculty of Science

Ataturk University

Erzurum, 25240

Turkey

e-mail: horhan@atauni.edu.tr 